\title{
Inhibition of Dopamine Receptor in Neonate Hippocampus: Immunolocalization of Post Synaptic Density Protein-95 and Dopamine Receptor in vivo
}

\author{
Babafemi J Laoye ${ }^{1 *}$, Oluwamolakun O Bankole ${ }^{1}$, Esther A Ekundayo ${ }^{1}$ and Azeez O Ishola ${ }^{2}$ \\ ${ }^{1}$ Department of Biological Sciences, College of Sciences, Afe Babalola University, Ado-Ekiti, Nigeria \\ ${ }^{2}$ Department of Anatomy, Neural Systems Unit, College of Medicine and Health Sciences, Afe Babalola University, Ado-Ekiti, Nigeria \\ "Corresponding author: Babafemi J Laoye, Department of Biological Sciences, College of Sciences, Afe Babalola University, Ado-Ekiti, Nigeria, Tel: \\ (+234)8136916125; E-mail: babafemif@abuad.edu.ng
}

Received date: May 05, 2018; Accepted date: May 25, 2018; Published date: June 01, 2018

Copyright: ( 2018 Laoye BJ, et al. This is an open-access article distributed under the terms of the Creative Commons Attribution License, which permits unrestricted use, distribution and reproduction in any medium, provided the original author and source are credited.

\begin{abstract}
The effect of haloperidol on neonatal dopaminergic neurotransmission in the hippocampus of postnatal day 20 rats (P.20) was investigated in this study. Haloperidol blocked dopamine receptors (D2R) and inhibited D2R on the membrane of neonate neurons. For this study the $0.5 \mathrm{ml}(20 \mathrm{mg} / \mathrm{kg})$ of haloperidol was administered to pregnant female animals intraperitoneally a week before delivery. At day P.20, 5 control animals and 5 haloperidol treated animals were taken to the behavioral studies room for the $Y$ maze and Novel object recognition test, which was done 7 am in the morning before mating. Electrophysiology was done with 2 control pups and 2 treated pups. Electrodes were implanted in the brain at the hippocampal region $2 \mathrm{~mm}$ beneath the bregma, $2 \mathrm{~mm}$ lateral to the midline. Anterior Posterior $(\mathrm{AP}=0)$, Medial Lateral $(\mathrm{ML}=2 \mathrm{~mm})$. Also immunolocalization and immunofluorescence of post synaptic density protein (PSD-95), hippocampal morphology and hippocampal neurons have been done respectively. Results from this study showed a decline in memory index for the $Y$ maze as a result of the effect of D2R blockade thereby inhibiting neurotransmission in newborns. Electrophysiology result in this study showed an increase in the root mean square (RMS) of control pups. The increase in RMS is equivalent to increase in wave burst pattern caused by neuronal excitation. Immunochemistry result showed an increase in the number of PSD-95 in the hippocampus of an increase in tyrosine hydroxylase in the hippocampus of the treated neonatal rats when compared to the control neonatal rats Immunofluorescence showed decline in the number of neurons in the haloperidol treated rats and it also caused hippocampal damage in terms of morphology. Furthermore, results from electrophysiology showed a statistical significant difference with $P$ value $0.04229(P<0.05)$ using the student $t$-test. These findings suggest that D2R inhibition may cause decline in memory function, impair learning in newborns and disrupt neonatal dopaminergic neurotransmission.
\end{abstract}

Keywords: Haloperidol; Bregma; PSD-95; D2R; RMS

\section{Introduction}

Dopamine Receptor (D2R) is a transmembrane receptor that is responsible for the uptake of dopamine which is a neurotransmitter secreted from the subtantia nigra in the mid brain for the purpose of cognition, coordination and memory function via neuron to neuron network [1]. Recent studies have shown that inhibition of D2R receptors in neonates causes neurological disorders and it is clearly visible in Parkinson's characterized by bradykinesia, tremor gait disorders etc. [2]. Also vesicular transport of D2R has been suspected to be involved in the cause of progression of $\mathrm{D} 2 \mathrm{R}$ related neurological disorders such as Parkinson's disease, schizophrenia and bipolar disorder [3]. Dopamine is a neurotransmitter that is secreted at the substatnsia nigra of the brain, which is responsible for movement, coordination and cognition [4]. It is an organic chemical of the catecholamine and phenethylamine families that plays several important roles in the brain and body. Its name is derived from its chemical structure: it is an amine synthesized by removing a carboxyl group from a molecule of its precursor chemical Levodopa (L-DOPA), which is synthesized in the brain and kidneys. It is also synthesized in plants and most multicellular animals [5]. In the brain, dopamine functions as a neurotransmitter a chemical released by neurons (nerve cells) to send signals to other nerve cells. The brain includes several distinct dopamine pathways, one of which plays a major role in reward-motivated behavior. Most 2 types of reward increase the level of dopamine in the brain, and most addictive drugs increase dopamine neuronal activity. Other brain dopamine pathways are involved in motor control and in controlling the release of various hormones. These pathways and cell groups form a dopamine system which is neuromodulatory [4]. Outside the central nervous system, it functions in several parts of the peripheral nervous system as a local chemical messenger. In blood vessels, it inhibits norepinephrine release and acts as a vasodilator (at normal concentrations); in the kidneys, it increases sodium excretion and urine output; in the pancreas, it reduces insulin production; in the digestive system, it reduces gastrointestinal motility and protects intestinal mucosa; and in the immune system, it reduces the activity of lymphocytes. With the exception of the blood vessels, dopamine in each of these peripheral systems is synthesized locally and exerts its effects near the cells that release it [3].

Several important diseases of the nervous system are associated with dysfunctions of the dopamine system, and some of the key medications used to treat them work by altering the effects of dopamine [4]. Parkinson's disease, a degenerative condition causing tremor and motor impairment, is caused by a loss of dopaminesecreting neurons in an area of the midbrain called the substantia nigra 
Citation: Laoye BJ, Bankole OO, Ekundayo EA, Ishola AO (2018) Inhibition of Dopamine Receptor in Neonate Hippocampus: Immunolocalization of Post Synaptic Density Protein-95 and Dopamine Receptor in vivo. Biol Med (Aligarh) 10: 441. doi: 10.4172/0974-8369.1000441

Page 2 of 6

[6]. Studies have shown that antipsychotics such as Haloperidol which causes dopamine receptor blockade may lead to Parkinsonism and progressively lead to Schizophrenia [7]. Levodopa is the most widely used treatment for the condition [5]. There is evidence that schizophrenia involves altered levels of dopamine activity, and most antipsychotic drugs used to treat this are dopamine antagonists which reduce dopamine activity. Similar dopamine antagonist drugs are also some of the most effective anti-nausea agents [5]. Restless legs syndrome and Attention Deficit Hyperactivity Disorder (ADHD) are associated with decreased dopamine activity [2]. Dopaminergic stimulants can be addictive in high doses, but some are used at lower doses to treat Attention Deficit Hyperactivity Disorder (ADHD). Dopamine itself is available as a manufactured medication for intravenous injection: although it cannot reach the brain from the bloodstream, its peripheral effects make it useful in the treatment of heart failure or shock, especially in newborn babies [2].

The aim of this study seeks to observe and examine D2R vesicle trafficking and docking on the membrane of neurons and PSD-95 scaffolding proteins in the developing mouse brain of P.20 after inhibiting D2R with Haloperidol and also to test for the effect of Haloperidol on memory function in neonates. Experimental Hypothesis: at the end of this study, we hypothesize that there would be a decline in memory function in the Wistar rats treated with haloperidol compared to the control group as a result of the blockage of $\mathrm{D} 2 \mathrm{R}$ receptors brought about by haloperidol which is a D2R blocker.

The specific objectives of this study are as follows: to compare the burst pattern of the neurons of P.20 neonates via electrophysiology and to determine the memory index of control and treated neonates via behavioural studies.

\section{Materials and Methods}

Sixteen (16) F1 Albino Wistar rats (12 females, 4 males) were purchased from the animal holding facility of Afe Babalola University Ado-Ekiti, Ekiti State, Nigeria. Haloperidol was purchased from Reich Pharmacy, Nigeria. Antibodies were purchased from ABCAM, USA.

\section{Animal preparation}

The animals were housed in the animal holding facility of Afe Babalola University, Nigeria. The animals were allowed to acclimatize to the new environment for 1 week. The animals were kept under alternating light and darkness with rat chow and water being given to the animals daily.

The albino Wistar rats were kept in separate cages, 4 animals per cage in 4 cages, having 3 females, one male in each cage. The animals were being placed in such manner for the purpose of mating.

All protocols were in accordance with the IACUC animal use guidelines and were approved by the Animal Use Ethical Committee of the Afe Babalola University, Nigeria.

\section{Mating}

Mating was done at the second week after acclimatization, 3 female albino Wistar rats and 1 male albino Wistar rat were placed in four different cages.

\section{Vaginal smear}

Vaginal smear was performed every morning for the female rats using the method of Marcondes et al. [8]. Pasteur pipette containing normal saline was dipped into the vagina of the female albino Wistar rat. The pipette was then used to suck up vaginal fluid. Two drops of the fluid which has the vagina fluid and normal saline was then placed on a glass slide and smeared. The smear was then observed underneath the microscope, using $10 \mathrm{X}$ objective. The essence of this was to observe the oestrus stage of the reproductive cycle of the albino rats. Any female animals which were observed to have reached the oestrus stage were separated to be alone with a male for constant mating. When the female animals were suspected to be pregnant, they were weighed and separated into different cages.

\section{Haloperidol administration}

The female animals were weighed and all the suspected pregnant animals (Treatment Group) were injected intraperitoneally with $0.5 \mathrm{ml}$ ( $20 \mathrm{mg} / \mathrm{kg}$ haloperidol for 7 days. Treatment was done every morning before the animals were fed with the rat chow.

\section{Behavioral tests}

Behavioral studies were done for the pups at P.20 after delivery. The animals were taken through the Novel Object Recognition test (NOR) and the Y maze test. The test was done for both control groups and treatment groups.

\section{Y Maze}

The Y Maze Test was done very early in the morning for both control and treatment groups pups at 7:30 am. 5 control and treatment pups (P.20). The test was done to assay for the spatial working memory index of the pups using the method of Adeniyi et al. [9] and each control animal was placed in the Y maze and was allowed to navigate and explore the 3 arms of the Maze for 3 minutes Observation of the exploratory movement was done using a camera connected to a laptop computer. The method used in this research is the method of Adeniyi et al. [9] and Onaolapo et al. [10].

After this the memory index was then calculated.

Memory index $=$ Number of right decisions $\div$ Total arm entries $-2 \times$ $100[9]$.

There is a -2 because the total number of arm entries of the Y maze is 3 , and the animal is expected to enter only one arm at once. This method is used by scientists all over the world for behavioral studies.

\section{NOR test}

The Novel object recognition test was done to assay for memory function. The test was done for both control and treatment groups. 5 animals each (control and treatment). For the first trial 2 identical objects were placed in a box and the animal was introduced into the box to familiarize itself with the objects for 5 minutes. After this, the second trial was done by replacing an identical object with an unidentical object. The test was done for both control and treatment group for 5 minutes and then the memory index was calculated.

Memory index $=$ Time spent on new object $\div$ Time spent on both new and old objects $\times 100[9]$. 
Citation: Laoye BJ, Bankole OO, Ekundayo EA, Ishola AO (2018) Inhibition of Dopamine Receptor in Neonate Hippocampus: Immunolocalization of Post Synaptic Density Protein-95 and Dopamine Receptor in vivo. Biol Med (Aligarh) 10: 441. doi: 10.4172/0974-8369.1000441

Page 3 of 6

\section{Electrophysiology}

Electrophysiology was performed with the use of fine electrodes and copper wire, which were inserted to the brain of 4 pups ( 2 control and 2 treated), $2 \mathrm{~mm}$ beneath the bregma, $2 \mathrm{~mm}$ lateral to the midline. Anterior Posterior $(\mathrm{AP}=0)$, Medial Lateral $(\mathrm{ML}=2 \mathrm{~mm})$. The animals were anaesthetized with ketamine. A small area on the scalp of the animals was shaved to expose the bregma and region above the orbit. A stereotaxic frame was used to hold the head of the animals in position to determine the location hippocampus $2 \mathrm{~mm}$ to the bregma. Hydrogen peroxide solution was used to clean the periosteum and blood markings on the scalp and a drill was made through the cranium using the coordinates. Then electrode was inserted on the $2 \mathrm{~mm}$ mark. The rubber base of the electrode was then sealed with the use of orthodontic resin and allowed to dry after which the spiker box was switched on to take electrophysiology readings on the computer.

Electrophysiology readings were taken after putting the animal to sleep with the use of $0.3 \mathrm{ml}$ ketamine. The immobile awake animal was then placed on a stereotaxic frame and electrodes connected to a spiker box were used to take brain readings from the Hippocampus using Audacity software and further analyzed in sigview software to get the root mean square and epoch readings in form of a wave curve [11].

\section{Immunohistochemistry (IHC)}

Two pups of both control and treatment groups were used for immunohistochemistry, the immobile awake animals were perfused with normal saline after anesthetizing the animal with $0.3 \mathrm{ml}$ of ketamine and then with formal saline. The purpose of this was to replace the blood with normal and formal saline to fix the brain tissue and clear the blood on the brain, to make it neat and beautiful for IHC. IHC was done with the use of anti PSD-95 and D2R anti body to label PSD-95. Immunofluorescence was done to map out the hippocampal neurons and the hippocampus with the use of vesicular GABA transporter (VGAT) and 4, 6-Diamidino-2-phenylindole (DAPI) respectively [12].

\section{Cell count}

Cell count of D2R receptors was performed using the image J software model IJ 1.46r. The number of D2R receptors in the control tissue was compared to that of the treated tissue [9].

\section{Results}

\section{Y maze test}

Spatial working memory was determined using Y-maze test. The percentage alternation which is the function of memory index in the Ymaze was determined to measure the spatial memory of the neonate animals after a 7-day treatment with haloperidol during last week of gestation. No significant statistical difference $(\mathrm{P}>0.05)$ was observed in the spatial memory (Memory Index) when the treatment groups were compared with the control although there was decrease in memory index of the treatment group when compared with the control.

\section{NOR test}

Novel object recognition test was done to measure non-spatial working memory of neonates (P.20) of animals with treatment of haloperidol and normal saline during last week of gestation for 7 days.
Neonates of haloperidol treated mothers showed decline in the memory index compared to control. There was no statistical significant difference between the groups $(\mathrm{P}>0.05)$ using $\mathrm{t}$-test even though the memory index of the treatment group was lower (Figure 1). (a) Y-Maze Test

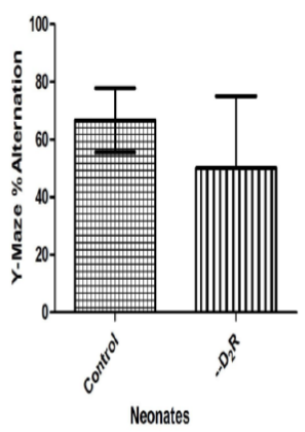

(b) Novel Object Recognition Test

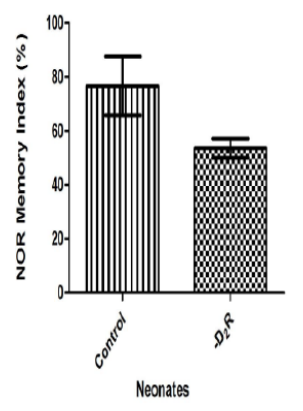

Figure 1: Motor-Cognitive function determined in Y-Maze test and NOR. (a) The total number of arm entries in the Y-Maze was determined to measure exploratory motor function associated with spatial memory after acute treatment duration. No significant change $(\mathrm{P}>0.05)$ was observed in exploratory motor function when the treatment groups were compared with the control. There was decrease in memory index of the treatment group when compared with the control which is shown on the graph. (b) For the Novel Object Recognition Test was used to determine non spatial working memory index of the neonates and results from the graph above showed a decline in memory index in neonates of haloperidol treated mothers but no statistical difference $(\mathrm{P}>0.05)$ was observed.

\section{Discussion}

Taken together, the outcome of this study confirms the role of dopaminergic receptor (D2R blockade and decline of Post synaptic density scaffolding proteins (D2R) in Parkinson's disease. Results gathered from this study revealed that D2R inhibition with the use of haloperidol on post-natal day 20 rats (P.20) revealed a decline in the memory index using Y-Maze [10]. But statistical analysis of the graph plotted revealed no statistical significance difference when comparing the haloperidol treated pups (P.20) together with the control pups with a $\mathrm{P}$ value greater than $0.05(\mathrm{P}>0.05)$ using the student $\mathrm{t}$-test to compare between one variable. This means that a level of $95 \%$ confidence interval there's no significance difference between the means of the control and treatment groups. Although from the graph (Figures 1a-1b) for Y maze test and NOR test [9] showed a decline in the memory index of the neonatal animals (P.20) when compared with the control. The results from the behavioral studies graph shows that haloperidol inhibition reduced the memory index in NOR and also the percentage alternation in the $\mathrm{Y}$ maze test. The results here infer that blocking neurogenesis in newborns would impair learning in newborns.

Furthermore, this study showed a decline in the PSD-95 cells (Post synaptic density proteins) from the cell count result in Figures 2 and 3a. The PSD-95 proteins are scaffolding proteins are proteins which are present in the membranes of dopaminergic neurons responsible for maintaining the integrity of the neuronal membrane, they provide 
Citation: Laoye BJ, Bankole OO, Ekundayo EA, Ishola AO (2018) Inhibition of Dopamine Receptor in Neonate Hippocampus: Immunolocalization of Post Synaptic Density Protein-95 and Dopamine Receptor in vivo. Biol Med (Aligarh) 10: 441. doi: 10.4172/0974-8369.1000441

Page 4 of 6

support together with the cytoskeletons such as F-actin filaments. Results from this study showed a decline in PSD-95 (Figure 3a) proteins and this affected the decline in memory index of the animals (P.20) which in turn impairs learning in newborns. The decline of PSD-95 associated with the treatment group (haloperidol treated P.20 pups) would lead to difficulty in the uptake of dopamine from neuron to neuron, thereby inhibit neurotransmission in newborns.

Furthermore, microscopic images taken to show localization of tyrosine hydroxylase (TH) showed increase of tyrosine hydroxylase in the hippocampus of the treated neonates when compared to the control (Figure 4). Looking at the graph in Figure 3b, the graph showed a great increase in $\mathrm{TH}$ in the hippocampus of treated rat newborns when compared with the control. This is expected because in this study, the central hypothesis suggests that D2R inhibition will increase NMDAR/AMPA dependent calcium signaling and this will in turn increase the rate of $\mathrm{D} 2 \mathrm{R}$ vesicle trafficking and docking on scaffolding proteins. As a result of $\mathrm{D} 2 \mathrm{R}$ inhibition, there would be an over expression of D2R in the hippocampus at P.20. The result from Figures $5 a-5 b$ is in line with the central hypothesis of this study, increase in $\mathrm{TH}$ is as a result of D2R blockade which would in the end lead to increase in the enzyme tyrosine hydroxylase (TH). The enzyme $\mathrm{TH}$ is responsible for catalyzing the conversion of L-tyrosine to L-3, 4dihydroxyphenylalanine (Levodopa). Levodopa is a precursor of dopamine. Because of the dopamine receptor inhibition there would be over expression of $\mathrm{D} 2 \mathrm{R}$ which eventually led to a decline in memory function of haloperidol treated animals (-D2R).
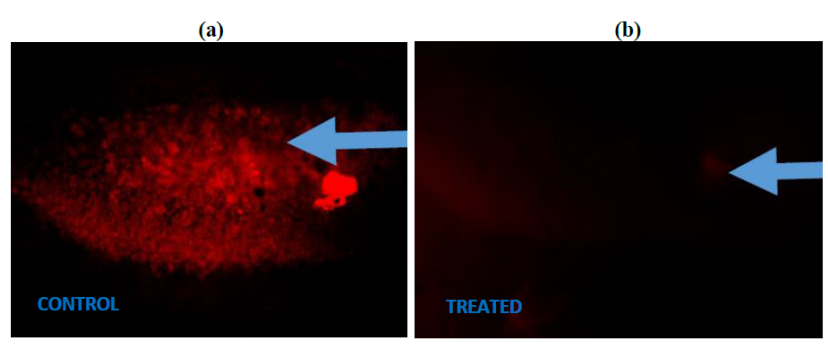

Figure 2: (a) Electrophysiology results showed epoch readings with an increase in root mean square (RMS) of the treatment group. Statistical analysis using the student t-test showed a significant difference $(\mathrm{P}<0.05)$. The increase in RMS which equates to the increase in burst pattern of the treatment P.20 pups might be due to D2R blockade with haloperidol which increased NMDAR/AMPA dependent calcium signaling by opening up calcium channels, thereby leading to increased and abnormal firing of neurons, because influx of calcium increased the action potential of the neurons, and in the end led to a decline in memory function of the neonates. (b) The epoch reading here showed reduction in burst pattern, the root mean square was reduced for the control was haloperidol wasn't administered. (a) PSD-95 Proteins (b)Tyrosine Hydroxylase Expression
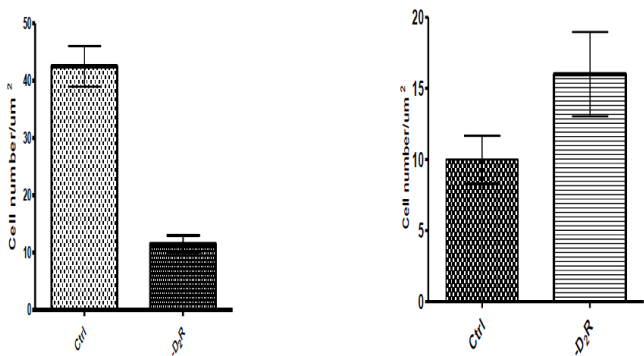

Figure 3: (a) The graph showed a decline in the PSD-95 cells (Post synaptic density proteins) from the cell count which was done at the hippocampus \{Cornus Ammonis (CA) and Dentate Gyrus (DG)\}. The PSD-95 proteins are scaffolding proteins are proteins which are present in the membranes of dopaminergic neurons responsible for maintaining the integrity of the neuronal membrane, they provide support together with the cytoskeletons such as F-actin filaments, newborns. The decline of PSD-95 associated with the treatment group (haloperidol treated P.20 pups) would lead to difficulty in the uptake of dopamine from neuron to neuron, thereby inhibit neurotransmission in newborns. (b) Microscopic images taken to show localization of tyrosine hydroxylase $(\mathrm{TH})$ showed increase of tyrosine hydroxylase in the hippocampus of the treated neonates when compared to the control. The graph showed a great increase in $\mathrm{TH}$ in the hippocampus of treated rat newborns when compared with the control.
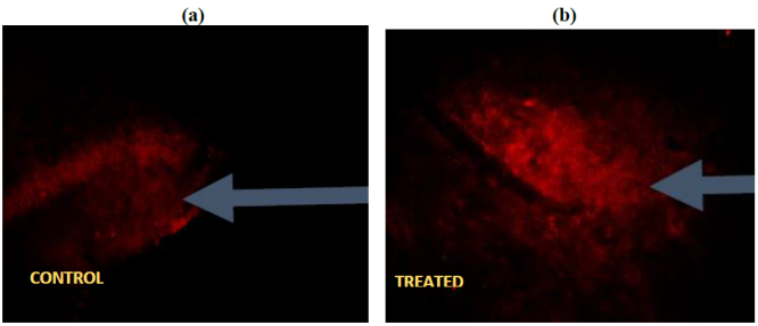

Figure 4: Microscopic image of rat P.20 neonate hippocampus (Control and Treated) showing tyrosine hydroxylase (red) (X60). The micrograph shows a great increase in $\mathrm{TH}$ in the hippocampus of treated rat newborns when compared with the control.

More so, electrophysiology results showed epoch readings with an increase in root mean square (RMS) of the treatment group. Statistical analysis using the student t-test showed a significant difference $(\mathrm{P}<0.05)$. The increase in RMS which equates to the increase in burst pattern of the treatment P.20 pups might be due to D2R blockade with haloperidol which increased NMDAR/AMPA dependent calcium signaling by opening up calcium channels, thereby leading to increased and abnormal firing of neurons, because influx of calcium increased the action potential of the neurons, and in the end led to a decline in memory function of the neonates (Figure 6). 
Citation: Laoye BJ, Bankole OO, Ekundayo EA, Ishola AO (2018) Inhibition of Dopamine Receptor in Neonate Hippocampus: Immunolocalization of Post Synaptic Density Protein-95 and Dopamine Receptor in vivo. Biol Med (Aligarh) 10: 441. doi: 10.4172/0974-8369.1000441

Page 5 of 6

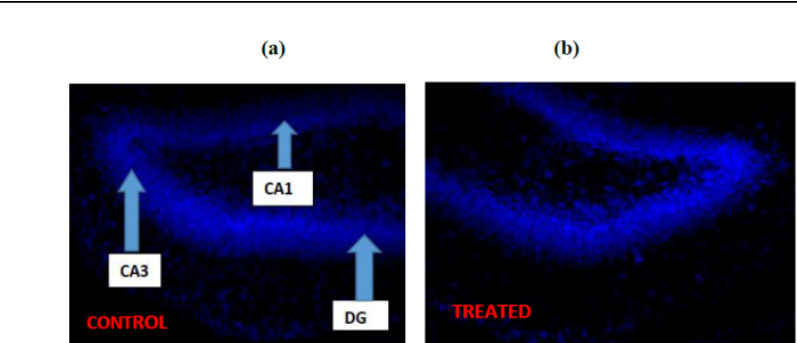

Figure 5: (a) The graph showed a decline in the PSD-95 cells (Post synaptic density proteins) from the cell count which was done at the hippocampus \{Cornus Ammonis (CA) and Dentate Gyrus (DG)\}. The PSD-95 proteins are scaffolding proteins are proteins which are present in the membranes of dopaminergic neurons responsible for maintaining the integrity of the neuronal membrane, they provide support together with the cytoskeletons such as F-actin filaments. Newborns: The decline of PSD-95 associated with the treatment group (haloperidol treated P.20 pups) would lead to difficulty in the uptake of dopamine from neuron to neuron, thereby inhibit neurotransmission in newborns. (b) Microscopic images taken to show localization of tyrosine hydroxylase (TH) showed increase of tyrosine hydroxylase in the hippocampus of the treated neonates when compared to the control. The graph showed a great increase in TH in the hippocampus of treated rat newborns when compared with the control.

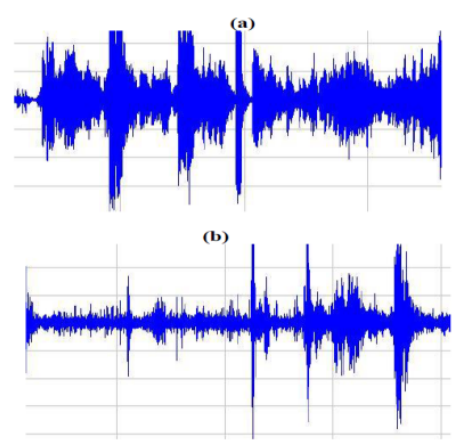

Figure 6: (a) Electrophysiology results showed epoch readings with an increase in root mean square (RMS) of the treatment group. Statistical analysis using the student t-test showed a significant difference $(\mathrm{P}<0.05)$. The increase in RMS which equates to the increase in burst pattern of the treatment P.20 pups might be due to D2R blockade with haloperidol which increased NMDAR/AMPA dependent calcium signaling by opening up calcium channels, thereby leading to increased and abnormal firing of neurons, because influx of calcium increased the action potential of the neurons, and in the end led to a decline in memory function of the neonates (b) The epoch reading here showed reduction in burst pattern, the root mean square was reduced for the control were haloperidol wasn't administered.

\section{Conclusion}

Inhibition of dopamine receptor D2R AND Post Synaptic Density Protein D2R showed a decline in memory function in cognition in neonates and it was associated with irregular burst pattern $\mathrm{s}$ via electrophysiology and decline in memory index via NOR and Y-maze behavioral study test. This study revealed via electrophysiology, immunolocalization and behavioral studies that D2R decline in memory function in neonates, and may equally lead to memory impairment which may concurrently lead to progressive Parkinsonism and Schizophrenia.

\section{Acknowledgements}

We appreciate the efforts of Mr Oludare and Mr. Oso for their help in caring for the animals and reagent preparations.

\section{Limitations}

For this study, funding was very difficult. The research was sponsored by the author and co-authors.

\section{Conflict of Interest}

The Authors hereby declare there is no conflict of interest associated with this study or any of the procedures and materials used for the purpose of the study.

\section{References}

1. Lemos JC, Friend DM, Kaplan AR, Shin JH, Rubinstein M, et al. (2016) Enhanced gaba transmission drives bradykinesia following loss of dopamine D2 receptor signaling. Neuron 4: 824-838.

2. Volkow ND, Wang GJ, Kollins SH, Wigal TL, Newcorn JH, et al. (2009) Evaluating dopamine reward pathway in ADHD: Clinical implication. JAMA 10: 1084-1091.

3. Shao L, Golbaz K, Honer WG, Beasley CL (2016) Deficits in axonassociated proteins in prefrontal white matter in bipolar disorder but not schizophrenia. Bipolar Disord 6: 58-60.

4. Lloyd K, Dayan P (2016) Safety out of control: Dopamine and defence. Behav Brain Funct 12: 1-15.

5. Tison F, Keywood C, Wakefield M, Durif F, Corvol JC, et al. (2016) A phase $2 \mathrm{~A}$ trial of the novel mGlur5-negative allosteric modulator dipraglurant for levodopa-induced dyskinesia in parkinson's disease. Mov Disord 23: 12-20.

6. Gonzalez R, Garitaonandia I, Poustovoitov M, Abramihina T, McEntire C, et al. (2016) Neural stem cells derived from human parthenogenetic stem cells engraft and promote recovery in a nonhuman primate model of parkinson's disease. Cell Transplant 16: 25-30.

7. Geetika K, Karen BC, José EL, Alexandra BN, Maria R, et al. (2016) Parkinsonism driven by antipsychotics originates from dopaminergic control of striatal cholinergic interneurons. Neuron 91: 67-78.

8. Marcondes FK, Bianchi FJ, Tanno AP (2002) Determination of the estrus phases cycle of rats: Some helpful considerations. Braz J Biol 62: 609-614.

9. Adeniyi PA, Ishola AO, Laoye BJ, Olatunji BP, Bankole OO, et al. (2016) Neural and behavioural changes in male periadolescent mice after prolonged nicotine-MDMA treatment. Metab Brain Dis 31: 93-107.

10. Onaolapo OJ, Onaolapo AJ, Mosaku TJ, Akanji OO, Abiodun OR (2012) Elevated plus maze and y-maze behavioral effects of sub chronic, oral low dose monosodium glutamate in Swiss albino mice. Journal of Pharmacy and Biological Sciences 3: 21-27.

11. Nagappa M, Netto AB, Taly AB, Kulkarni GB, Umamaheshwara Rao GS, et al. (2016) Electrophysiological observations in critically ill GuillainBarre syndrome. Neurol India 5: 914-920.

12. Duncan SM, Seigel GM (2016) High-contrast enzymatic immunohistochemistry of pigmented tissues. J Biol Methods 3: 3-10. 